\title{
A Prospective Study Evaluating Sleep Quality and Disorders in Post-ARDS Patients
}

\author{
Juvva Kishan Srikanth ${ }^{1} \cdot$ Rohit Kumar ${ }^{1}$ - Neeraj Kumar Gupta ${ }^{1}$ Pranav Ish ${ }^{1}$. Siddharth Raj Yadav ${ }^{1}$. \\ Shibdas Chakrabarti ${ }^{1} \cdot$ Nitesh Gupta $^{1}$
}

Received: 27 April 2021 / Revised: 27 April 2021 / Accepted: 25 July 2021 / Published online: 30 July 2021

(c) The Author(s), under exclusive licence to Springer Nature Singapore Pte Ltd. 2021

\begin{abstract}
Introduction Critically ill patients are predisposed to developing sleep disorders due to multiple factors like pre-existing sleep disorders, severe acute illness, sleep-altering medical interventions, and the disturbing intensive care unit (ICU) environment. In the current study, a multi-modality approach has been attempted to capture the different aspects of sleep disturbances, including insomnia (using ISI), daytime sleepiness (using ESS), sleep quality (using PSQI), sleep architecture, and SDB (using PSG).

Materials and Methods The eligible ARDS survivor patients were updated about the study's design prior to hospital discharge. At admission, data regarding demographic details, clinical history, etiology of ARDS, and $\mathrm{PaO}_{2} / \mathrm{FiO}_{2}(\mathrm{P} / \mathrm{F})$ ratio at presentation, Acute Physiology and Chronic Health Evaluation II (APACHE II) and Sequential Organ Failure Assessment (SOFA) scores was collected. All enrolled patients were evaluated twice (early-within 7 days of admission and late-after 6 weeks of discharge) by the Richards-Campbell Sleep Questionnaire (RCSQ), Insomnia severity index (ISI), and level 1 PSG. Additionally, ESS questionnaire and the Pittsburgh Sleep Quality Index (PSQI) were also recorded in late evaluation. Abnormal sleep was defined if one or more of the following characteristics met: ISI $>15$, ESS $>10$, global PSQI $>5$, AHI $\geq 5$ events/h.

Results Thirty patients were recruited out of the total of 88 ARDS patients screened at admission. The median (IQR) $\mathrm{PaO}_{2} /$ $\mathrm{FiO}_{2}$ ratio and APACHE II scores were 176 (151-191.5) and 14 (14-16), respectively. The median (IQR) duration of stay in the ICU was 10 (7.3-19.5) days. The median RCSQ score in the early and late evaluation was 42 and 69 , respectively. The mean ISI score in the early evaluation was $16.67 \pm 4.72$, which decreased to $11.70 \pm 5.03$ in late evaluation $(p<0.05)$. ISI score $>15$ (clinical insomnia) was found in 18 out of 30 subjects (60\%) in early evaluation and 11 out of $30(36 \%)$ in late evaluation. During the early evaluation, sleep efficiency was low (median 59.9\% and predominantly N1 and N2) which improved in late evaluation (median 80.6\%). Of the 30 patients, only 4 had AHI $>5$ in early evaluation and none in late evaluation. Neither of P/F ratio, SOFA, and APACHE II scores did correlate with ICU events in the early and late evaluations. Regression analysis showed subjects with ICU stay more than 10 days, duration of IMV more than 7 days, Fentanyl more than $7 \mathrm{mg}$, duration of sedative use more than 7 days was independently associated with poor objective sleep quality (low sleep efficiency, low TST and high arousal index) during the early and late evaluations after ICU discharge compared to counterparts ( $p$ value $<0.05$ ).

Conclusion We conclude that sleep quality in ARDS survivors was poor within 7 days of ICU discharge, characterized by severe disruption of sleep architecture and sleep-disordered breathing. After 6 weeks of ICU discharge sleep quality showed significant improvement in the N3 stage and AHI, however persistent insomnia was observed even at 6 weeks. Therefore, prior identification of risk factors and early diagnosis of sleep quality disorders in post-ARDS patients is essential.
\end{abstract}

Keywords Respiratory distress syndrome/complications · Polysomnography $\cdot$ Intensive care units $\cdot$ Sleep initiation and maintenance disorders $\cdot$ Sleep apnea syndromes $\cdot$ Severity of illness index

Nitesh Gupta

niteshgupta2107@gmail.com

Extended author information available on the last page of the article 


\section{Introduction}

Sleep is critical for the health and wellbeing of an individual. High-quality and efficient sleep of adequate duration helps consolidate memory, regulate the immune system, and coordinate neuroendocrine function [1-3]. Abnormalities in sleep profile and duration will increase the risk of a various broad range of adverse health effects, including cardiovascular disease, depression, cognitive impairment, seizures, and eventually mortality [4-8].

Critically ill patients are predisposed to developing sleep disorders due to multiple factors like pre-existing sleep disorders, severe acute illness, sleep-altering medical interventions, and the disruptive intensive care unit (ICU) environment [9]. In critically ill patients, there are multiple factors predisposed to above-like environmental factors such as noise, light, and care activities and treatment-related factors like sedatives [10]. Moreover, patients with acute respiratory distress syndrome (ARDS) are predisposed to the risk of patient-ventilator dyssynchrony and frequently require sedatives and other psychotropic medications. All these factors make them a unique population, especially prone to sleep disturbances [11].

The primary objective of this study was to determine the prevalence of sleep disturbances diagnosed based on PSG in patients who recovered from ARDS early (less than 7 days) and late periods (more than 6 weeks) after ICU discharge.

\section{Materials and Methods}

The current study was a prospective, observational study conducted over 18 months in the Department of Pulmonary, Critical Care and Sleep Medicine at a center of excellence in India.

\subsection{Study Population}

Patients were included in the study if they satisfied all the following criteria: (1) age 18-65 years. (2) ARDS defined by the Berlin Definition, 2012. (3) ICU admission of at least $48 \mathrm{~h}$ duration. The exclusion criteria were: (1) patients with pre-existing neurological, psychiatric, and cognitive disorders. (2) Diagnosed with sleep disorders. (3) Tracheostomized. (4) Receiving any sedative or psychotropic medication after discharge from ICU. (5) Chronic obstructive pulmonary disease (COPD) and congestive heart failure. (6) Pregnancy. (7) Mechanical ventilation and ICU admission in the preceding 6 months.

\subsection{Methodology}

Eligible ARDS survivor patients were updated about the study's design prior to hospital discharge. If the patients agreed to participate in the study, an assessment in sleep laboratory within 7 days (first evaluation), and another after 6 weeks (second evaluation) of ICU discharge were done. All the consecutive patients meeting inclusion criteria were recruited as per protocol. The first evaluation described as "early," and the second evaluation described as "late" in the current manuscript. The ICU comprising of ten beds, had a nurse: patient ratio of 1:2. All patients gave informed consent, and the Institutional Ethical Committee approved the study protocol.

\subsection{Evaluation of Patients at Baseline}

At admission, data comprised demographic details (age/sex/ body mass index), clinical history, etiology of ARDS, and $\mathrm{PaO}_{2} / \mathrm{FiO}_{2}(\mathrm{P} / \mathrm{F})$ ratio at presentation. The Acute Physiology and Chronic Health Evaluation II (APACHE II) and Sequential Organ Failure Assessment (SOFA) scores were recorded (higher scores associated with a worse outcome).

All patients underwent appropriate laboratory investigations and therapeutic management as per accepted protocols. The duration of mechanical ventilation (in days), ICU stay and the dose and duration of sedatives and neuromuscular blockers recorded - the patient recruitment and evaluation presented in Table 1.

\subsection{Measurements of Sleep Quality and Quantity}

All enrolled patients were evaluated twice, both during early and late periods, by the Richards-Campbell Sleep Questionnaire (RCSQ), insomnia severity index (ISI), and level 1 PSG. After 6 weeks of ICU discharge, patients were additionally evaluated by the ESS questionnaire and the Pittsburgh Sleep Quality Index (PSQI). The profile of sleep disorders was recorded based on the questionnaires and PSGs at two different time points, "early" and "late", after ICU stay.

\subsection{Polysomnography}

Level 1 polysomnography (PSG) was performed using Alice 6 L.D.X. (Philips Respironics, USA) in the dedicated sleep laboratory. Level 1 PSG includes monitoring of the electroencephalogram (EEG) using two frontal, two central, and two occipital channels (placed according to the international 10-20 system), electrooculogram (EOG), submental and anterior tibialis, electromyogram (EMG), and electrocardiogram (ECG) (lead I). Additionally, it includes the chest and abdominal movements, airflow (using oronasal thermal 
Table 1 Baseline characteristics of the study population

\begin{tabular}{ll}
\hline Characteristics & Value \\
\hline Age, in years & 33.50 (mean) (21-59) \\
Gender (female:male) & $1.3: 1$ \\
BMI (mean \pm SD) & $23.50 \pm 3.02$ \\
Diabetes mellitus & $3(10 \%)$ \\
Hypertension & $4(13 \%)$ \\
Etiology & \\
Acute febrile illness of unknown etiology & $10(33.33)$ \\
Acute pancreatitis & $1(3.3)$ \\
Aspiration pneumonia & $2(6.7)$ \\
Community-acquired pneumonia & $5(16.7)$ \\
Enteric fever & $1(3.3)$ \\
Miliary tuberculosis & $7(23.3)$ \\
Postoperative MODS & $1(3.3)$ \\
Scrub typhus & $2(6.7)$ \\
Acute febrile illness (Malaria) with MODS & $1(3.3)$ \\
ICU characteristics & \\
Duration of ICU (in days) (median) & 13.50 \\
Duration of IMV (in days) (median) & 10 \\
P/F ratio (median) & 139 \\
SOFA Score (median) & 7 \\
APACHE-II Score (median) & 18 \\
Septic shock & $9(30 \%)$ \\
Cumulative doses of sedatives & \\
Midazolam (in mg) & 80 \\
Fentanyl (in mg) & 8 \\
Morphine (in mg) & 1.50 \\
Duration of sedative use (in days) & 6 \\
Doses of vasopressors & \\
Noradrenaline dose (in mg) & \\
Doses of Neuro Muscular Blockers & \\
Vecuronium (in mg) & \\
Duration of vecuronium use (in days) & \\
\hline & \\
\hline
\end{tabular}

MODS multi organ dysfunction syndrome, $B M I$ body mass index, $I C U$ intensive care unit, $I M V$ invasive mechanical ventilation, SOFA sequential organ failure assessment, APACHE II acute physiology and chronic health evaluation II

sensor and nasal air pressure transducer), and pulse oximetry. Sleep stages and events scored per the 2017 American Academy of Sleep Medicine criteria [12]. The total sleep time (TST), sleep efficiency, sleep latency, wake after sleep onset (WASO), duration of each sleep stage, and arousal index calculated. Sleep-disordered breathing (SDB) was defined by apnea-hypopnea index (AHI) $\geq 5$ events/h with symptoms or AHI $\geq 15$ events/h without symptoms. Abnormal sleep was defined in the event of one or more of the following characteristics: ISI $>15$, ESS $>10$, global PSQI $>5, \mathrm{AHI} \geq 5$ events/h.

\subsection{Statistical Analysis}

The continuation data were expressed as mean or median (inter-quartile range (IQR); categorical data were expressed as a number (percent). The mean and standard deviations of the measurements per group were calculated. The difference between the two groups was determined using a student $t$ test, and the level of significance was set at $p<0.05$. Pearson correlation was done for ICU events and determined sleep disorders. Logistic regression analysis was performed to assess predictors of poor sleep after ICU stay. The analysis was performed via statistical analysis Software (SPSS 22.00 for windows; S.P.S.S.inc, Chicago, USA).

\section{Results}

Thirty patients were recruited from a total of 88 ARDS patients screened at admission (Fig. 1). Acute febrile illness of unknown etiology with multi-organ dysfunction syndrome was the commonest etiology for ARDS. The median (IQR) $\mathrm{PaO}_{2} / \mathrm{FiO}_{2}$ ratio and APACHE II scores were 176 (151-191.5) and 14 (14-16), respectively. The median (IQR) duration of stay in the ICU was 10 (7.3-19.5) days. Midazolam (14 [70\%] patients) and vecuronium (12 [60\%] patients) were the most commonly used sedative and neuromuscular blocking agents, respectively (Table 1).

The median RCSQ score in the early evaluation was 42 , indicating a poor sleep quality. The score increased to 69 in late evaluation, signifying a subjective improvement in sleep quality over a period $(p<0.05)$. The mean ISI score in the early evaluation was $16.67 \pm 4.72$, which decreased to $11.70 \pm 5.03$ in late evaluation $(p<0.05)$. ISI score $>15$ (clinical insomnia) was found in 18 out of 30 subjects (60\%) in early evaluation and 11 out of $30(36 \%)$ in late evaluation.

During the early evaluation, sleep efficiency was low (median 59.9\%) and predominantly comprised of N1 and $\mathrm{N} 2$ stages. In late evaluation, notable improvement in sleep efficiency (median 80.6\%) was documented. Of 30 patients, only four had AHI $>5$ (SDB) during the early evaluation and none in late evaluation. Ten $(33.3 \%)$ patients had a global PSQI above the threshold score of 5, representing low overall sleep quality (Table 2). The ESS score documented as a median [IQR] score of 6 [3.3-7] (Table 3).

Correlation between ICU events and the sleep abnormalities in early and late evaluations after ICU discharge is shown in Tables 4 and 5. P/F ratio, SOFA, and APACHE II scores did not correlate with ICU events in the early and late evaluations. Regression analysis showed subjects with ICU stay more than 10 days, duration of IMV more than 7 days, Fentanyl more than $7 \mathrm{mg}$, duration of sedative use more than 7 days was independently associated with poor objective sleep quality (low sleep efficiency, low TST and high 
Fig. 1 The flow diagram of study participants

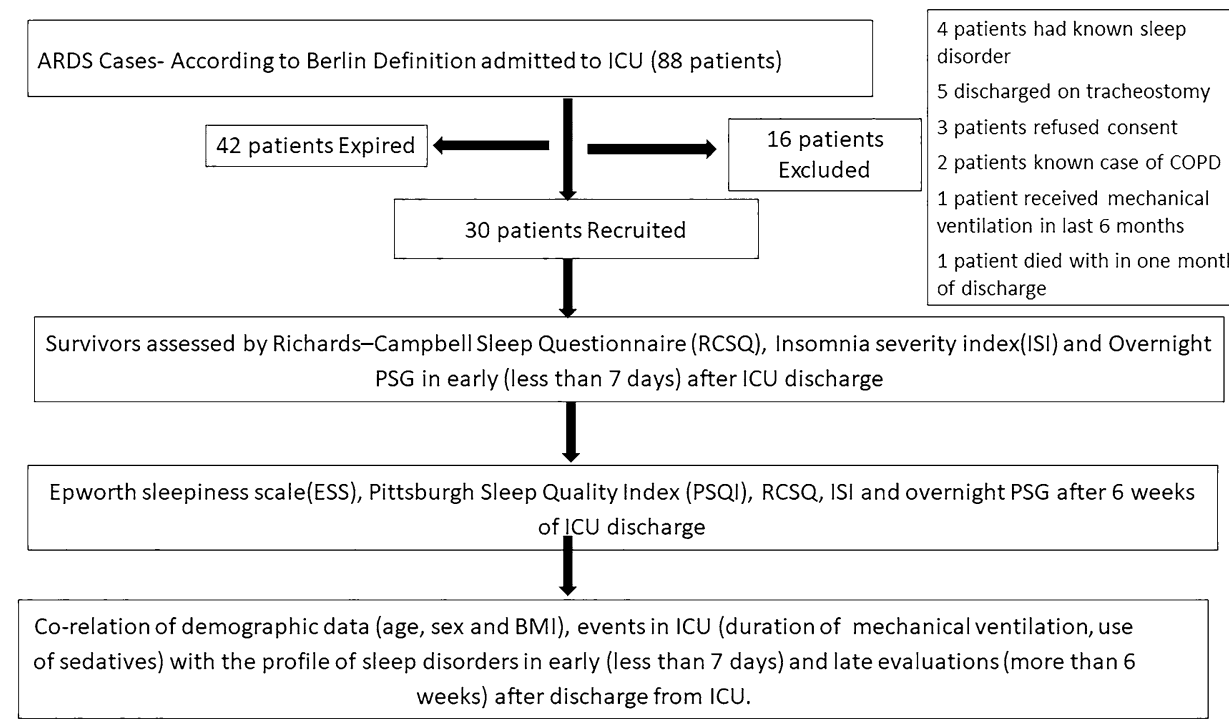

Table 2 Measures of sleep quality in study subjects

\begin{tabular}{|c|c|c|c|}
\hline Score & $\begin{array}{l}\text { Early (within } 7 \text { days after ICU } \\
\text { discharge) }\end{array}$ & $\begin{array}{l}\text { Late (after } 6 \text { weeks of ICU } \\
\text { discharge) }\end{array}$ & $p$ value \\
\hline Richards-Campbell Sleep Questionnaire Score & 42 & 69 & $<0.01$ \\
\hline $\begin{array}{l}\text { Epworth Sleepiness Score (median) } \\
\text { Number of patients with Epworth Sleepiness Score }>10\end{array}$ & NA & $\begin{array}{l}4(3-7) \\
0\end{array}$ & NA \\
\hline Pittsburgh Sleep Quality Index $>5, n(\%)$ & NA & $10(33.33 \%)$ & NA \\
\hline Insomnia Severity Index Score & 17 & 12 & $<0.01$ \\
\hline
\end{tabular}

NA not applicable

Table 3 Polysomnographic characteristics in the study participants

\begin{tabular}{llll}
\hline Variables & $\begin{array}{l}\text { Early (within 7 days after } \\
\text { ICU discharge) }\end{array}$ & $\begin{array}{l}\text { Late (more than 6 weeks after } \\
\text { ICU discharge) }\end{array}$ & $p$ value \\
\hline Total sleep time (in min) & $239.50(193.9-309.1)$ & $310.84(297.9-338.4)$ & $<0.01$ \\
Sleep latency (in min) & 24 & 13 & $<0.01$ \\
WASO (in min) & 124 & 64.50 & $<0.01$ \\
Sleep efficiency, \% of TST & 59.95 & 80.06 & $<0.01$ \\
Sleep stages & & & $<0.01$ \\
N1 (in min) & 48.84 & 15.26 & $<0.01$ \\
N2 (in min) & 122.54 & 161.84 & $<0.01$ \\
N3 (in min) & 20.90 & 49.55 & $<0.01$ \\
REM (in min) & 43.49 & 84.85 & \\
AHI [Median (IQR)] & $3(2-7)$ & $3(2-4)$ & $<0.01$ \\
Number of patients with AHI $>5$, & $4(13.3 \%)$ & 0 & \\
$n$ (\%) & & 20 & \\
Arousal Index & 27 & &
\end{tabular}

All values are median (IQR) or number (\%)

$A H I$ apnea-hypopnea index, REM rapid eye movement, IQR interquartile range, WASO wake after sleep onset 
Table 4 Significant correlation between ICU events with the determined sleep abnormalities in early (less than 7 days after ICU discharge)

\begin{tabular}{|c|c|c|c|c|}
\hline & ISI score & Sleep latency & AHI & Arousal index \\
\hline \multicolumn{5}{|c|}{ Duration of ICU (in days) } \\
\hline$R$ & $0.457^{*}$ & $0.409 *$ & $0.538 * *$ & $0.409 *$ \\
\hline$p$ & 0.011 & 0.025 & 0.002 & 0.025 \\
\hline \multicolumn{5}{|c|}{ Duration of IMV (in days) } \\
\hline$R$ & $0.523 * *$ & $0.447 *$ & $0.579 * *$ & $0.447 *$ \\
\hline$p$ & 0.003 & 0.013 & 0.001 & 0.013 \\
\hline \multicolumn{5}{|c|}{ Noradrenaline dose (in mg) } \\
\hline$R$ & $0.461^{*}$ & 0.168 & $0.377^{*}$ & 0.168 \\
\hline$p$ & 0.010 & 0.374 & 0.040 & 0.374 \\
\hline \multicolumn{5}{|c|}{ Muscle relaxants (vecuronium in $\mathrm{mg}$ ) } \\
\hline$R$ & $0.442 *$ & 0.251 & 0.323 & 0.251 \\
\hline$p$ & 0.014 & 0.181 & 0.082 & 0.181 \\
\hline \multicolumn{5}{|c|}{ Duration of vecuronium use (in days) } \\
\hline$R$ & $0.525^{* *}$ & 0.250 & $0.364 *$ & 0.250 \\
\hline$p$ & 0.003 & 0.183 & 0.048 & 0.183 \\
\hline \multicolumn{5}{|c|}{ Midazolam (in mg) } \\
\hline$R$ & $0.522 * *$ & 0.337 & $0.645^{* *}$ & 0.337 \\
\hline$p$ & 0.003 & 0.069 & 0.000 & 0.069 \\
\hline \multicolumn{5}{|c|}{ Fentanyl (in mg) } \\
\hline$R$ & $0.513 * *$ & $0.386^{*}$ & $0.459^{*}$ & $0.386^{*}$ \\
\hline$p$ & 0.004 & 0.035 & 0.011 & 0.035 \\
\hline \multicolumn{5}{|c|}{ Duration of sedative use (in days) } \\
\hline$R$ & $0.617 * *$ & $0.522 * *$ & $0.887 * *$ & $0.522 * *$ \\
\hline$p$ & 0.000 & 0.003 & 0.000 & 0.003 \\
\hline
\end{tabular}

$I C U$ intensive care unit, IMV invasive mechanical ventilation, ISI Insomnia Severity Index Score, $A H I$ apnea hypopnea index, $R$ correlation coefficient, $p p$ value

*Correlation is significant at level 0.05 ; $*$ Correlation is significant at level 0.01

arousal index) during the early and late evaluations after ICU discharge compared to counterparts ( $p$ value $<0.05$ ).

\section{Discussion}

In the current study, a multi-modality approach has been attempted to capture the different aspects of sleep disturbances, including insomnia (using ISI), daytime sleepiness (using ESS), sleep quality (using PSQI), sleep architecture, and SDB (using PSG). To the best of our knowledge, this is the first study in which an exclusive cohort of ARDS patients has been evaluated for sleep disturbances over different periods after ICU stay using level 1 PSG.

In the present study, subjective sleep disturbance was pronounced, similar to previous studies in ICU survivors [13]. The prevalence of ISI-defined clinical insomnia at early evaluation in the present study was $36 \%$, similar to the prevalence at 3-months of ICU discharge reported in the literature [14]. Insomnia after ICU stay can be attributed to environmental factors in ICU (noise, light) and patient care activities (procedures, medications) [15]. The present study documented improvement in insomnia after discharge. Thus, extra attention should be given to screening for insomnia during discharge and follow-up of ARDS survivors, affecting life quality.

One third of patients had global PSQI $>5$ in the late evaluation. This is similar to the study by Solverson et al. [16], which showed $62 \%$ of patients scored above the PSQI threshold after 3 months of ICU discharge. The higher incidence of poor sleep quality in the above study can be due to multiple comorbidities, including polytrauma and surgical patients, in the study population. We also observed low sleep efficiency (median 59\%) in early evaluation, which is similar to previous studies [17]. Sleep efficiency improved in late evaluation but did not reach the reported normal population average values.

The current study shows the increased duration of N1, and reduced N3 and REM sleep in early evaluation on evaluating sleep architecture. Increased duration of N1 suggests sleep fragmentation caused by sleep disturbance. Reduced duration of N3 sleep and REM indicates a lack of deep sleep, which are the restorative sleep stages for memory formation [18]; poor sleep quality might contribute to long-term cognitive dysfunction. The sleep architecture showed a trend towards improvement in stages of sleep-in late evaluation. Similarly, Dhooria et al. [17] showed low percentages of N3 and REM sleep within 1 month of discharge from ICU. The study by Alexopoulou et al. [19] reported improved sleep architecture (significant increase in N3 stage) 6 months after hospital discharge in general critically ill patients.

Sleep latency in the early and late evaluations after ICU discharge positively correlated with duration of ICU (in days), duration of IMV (in days) and duration of sedatives. Poor sleep quality and architecture after ICU discharge attributed to mechanical ventilation, benzodiazepine, opioids, norepinephrine, or epinephrine [20]. So from the above findings, it can be said that ARDS survivors exhibit sustained long-term poor sleep quality, which needs further confirmation in a large study cohort for more extended periods.

Of 30 patients, only 4 had $\mathrm{AHI}>5$ in early evaluation and none in late evaluation. In contrast, Dhooria et al. [17] documented 3 of 20 ARDS survivors had AHI $>5$ events/h, and the rest had AHI $<5$ events/h in PSG less than 1 month after ICU discharge. However, subsequent follow up of patients was not done.

In contrast, Chishti et al. [21] reported $73 \%$ of patients had AHI $>5$ events/h. The plausible explanation for the discrepancy in studies of ICU survivors, it is not possible to ascertain whether the SDB was pre-existing or appeared 
Table 5 Significant correlation between ICU events with the determined sleep abnormalities in late (after 6 weeks of ICU discharge)

\begin{tabular}{|c|c|c|c|c|c|}
\hline Parameter & ESS & ISI & PSQI & Sleep latency & Arousal index \\
\hline \multicolumn{6}{|c|}{ Duration of ICU (in days) } \\
\hline$r$ & -0.107 & $0.362 *$ & 0.286 & $0.409 *$ & $0.444 *$ \\
\hline$p$ & 0.573 & 0.049 & 0.125 & 0.025 & 0.014 \\
\hline \multicolumn{6}{|c|}{ Duration of IMV (in days) } \\
\hline$R$ & -0.065 & $0.409 *$ & 0.344 & $0.447 *$ & $0.497 * *$ \\
\hline$p$ & 0.732 & 0.025 & 0.062 & 0.013 & 0.005 \\
\hline \multicolumn{6}{|c|}{ Noradrenaline dose (in mg) } \\
\hline$R$ & -0.270 & $0.401 *$ & $0.458^{*}$ & 0.168 & 0.215 \\
\hline$p$ & 0.148 & 0.028 & 0.011 & 0.374 & 0.255 \\
\hline \multicolumn{6}{|c|}{ Muscle relaxants (vecuronium in $\mathrm{mg}$ ) } \\
\hline$R$ & -0.217 & $0.381 *$ & $0.434^{*}$ & 0.251 & 0.286 \\
\hline$p$ & 0.249 & 0.038 & 0.016 & 0.181 & 0.125 \\
\hline \multicolumn{6}{|c|}{ Duration of muscle relaxants use (in days) } \\
\hline$R$ & -0.197 & $0.477 * *$ & $0.467 * *$ & 0.250 & 0.250 \\
\hline$p$ & 0.297 & 0.008 & 0.009 & 0.183 & 0.183 \\
\hline \multicolumn{6}{|c|}{ Midazolam (in mg) } \\
\hline$R$ & -0.029 & $0.450 *$ & $0.605 * *$ & 0.337 & 0.318 \\
\hline$p$ & 0.880 & 0.013 & 0.000 & 0.069 & 0.087 \\
\hline \multicolumn{6}{|c|}{ Fentanyl (in mg) } \\
\hline$R$ & -0.082 & $0.440^{*}$ & 0.266 & $0.386^{*}$ & $0.405^{*}$ \\
\hline$p$ & 0.665 & 0.015 & 0.155 & 0.035 & 0.027 \\
\hline \multicolumn{6}{|c|}{ Duration of sedative use (in days) } \\
\hline$R$ & 0.128 & $0.555^{* *}$ & $0.641 * *$ & $0.522 * *$ & $0.466 * *$ \\
\hline$p$ & 0.499 & 0.001 & 0.000 & 0.003 & 0.010 \\
\hline
\end{tabular}

$I C U$ intensive care unit, IMV invasive mechanical ventilation, ISI Insomnia Severity Index Score, ESS Epworth Sleepiness Score, PSQI Pittsburgh Sleep Quality Index, $R$ correlation coefficient, $p p$ value *Correlation is significant at level 0.05 ; **Correlation is significant at level 0.01 after the acute illness. Furthermore, the prevalence of SDB is higher with increasing age and BMI. The mean age in Chishti et al. study was 63 years, which can cause higher SDB prevalence. In the present study, both the median age (33 years) and the median BMI $\left(27.0 \mathrm{~kg} / \mathrm{m}^{2}\right)$ of patients were low, and we had excluded the subjects with prior history of sleep disorders.

In the current study, AHI in the early period positively correlated with the duration of ICU (in days), duration of IMV (in days). Further, those with AHI > 5 events/h had a longer duration and higher sedative use dose. The possible explanation for the above finding would be longer the duration of ICU stay, more is the chance of ICU-acquired weakness. Thus, the pharyngeal dilators, particularly the genioglossus, reduce the muscles' responsiveness to negative intra-luminal airway pressure, leading to obstructive events [22].

In the current study, the $\mathrm{P} / \mathrm{F}$ ratio, SOFA score, and APACHE-II score did not correlate with any of the determined sleep disorders among early and late evaluations after ICU discharge. Notably, in the present study, the severity of illness at admission or during ICU stay does not determine the sleep quality after discharge from ICU. Alexopoulou et al. reported in multiple regression analysis APACHE II as the only variable that was negatively related to sleep efficiency [18].

The regression analysis to find predictors of poor sleep quality in ARDS patients after ICU discharge revealed that ICU duration more than 10 days, mechanical ventilation more than 7 days, fentanyl use of more than $7 \mathrm{mg}$, and sedative use of more than 7 days foretells a more inferior sleep quality after ICU discharge.

The current study's strengths are the prospective collection of data from ARDS patients and objective assessment of sleep quality by level 1 PSG (Gold standard) at two different periods after ICU discharge. Additional strengths comprise excluding patients with pre-existing sleep disorders by history or previous hospital records to minimize the bias.

The present study's limitations include small sample size and using ISI to diagnose insomnia rather than a standardized clinical interview. Notably, the current study population primarily consists of predominantly young individuals 
with ARDS. Thus, the results may not hold to other ICU survivors. Correspondingly, the nursing care activities, environment of ICU (light, noise and sound) were not recorded as factors disturbing sleep. Our findings need replication in more extensive and more diverse samples of ARDS survivors.

\section{Conclusion}

The present study gives insight into sleep quality in ARDS survivors and identifies which patients are at the highest risk for poor sleep quality, considering interventions in the ICU or post-discharge to improve sleep and overall recovery. Therefore, prior identification of risk factors and early diagnosis of sleep quality disorders in post-ARDS patients is essential.

Author Contributions All the seven authors contributed to-substantial contributions to the conception or design of the work; or the acquisition, analysis, or interpretation of data for the work; and drafting the work or revising it critically for important intellectual content; and final approval of the version to be published; and agreement to be accountable for all aspects of the work in ensuring that questions related to the accuracy or integrity of any part of the work are appropriately investigated and resolved.

Funding Nil.

\section{Declarations}

Conflict of interest There are no conflicts of interest.

\section{References}

1. Stickgold R. Neuroscience: a memory boost while you sleep. Nature. 2006;444:559-60.

2. Ibarra-Coronado E, Pantaleón-Martínez A, Velazquéz-Moctezuma J, Prospéro-García O, Méndez-Díaz M, Pérez-Tapia M, et al. The bidirectional relationship between sleep and immunity against infections. J Immunol Res. 2015;2015:1-14.

3. Pisani MA, Friese RS, Gehlbach BK, Schwab RJ, Weinhouse GL, Jones SF. Sleep in the intensive care unit. Am J Respir Crit Care Med. 2015;191:731-8.

4. Grandner MA, Sands-Lincoln MR, Pak VM, Garland SN. Sleep duration, cardiovascular disease, and proinflammatory biomarkers. Nat Sci Sleep. 2013;5:93-107.

5. Samsonsen C, Sand T, Brathen G, Helde G, Brodtkorb E. The impact of sleep loss on the facilitation of seizures: a prospective case crossover study. Epilepsy Res. 2016;127:260-6.
6. Li L, Wu C, Gan Y, Qu X, Lu Z. Insomnia and the risk of depression: a meta-analysis of prospective cohort studies. BMC Psychiatry. 2016;16:375.

7. Gallicchio L, Kalesan B. Sleep duration and mortality: a systematic review and meta-analysis. J Sleep Res. 2009;18:148-58.

8. Malow BA. Sleep deprivation and epilepsy. Epilepsy Curr. 2004;4:193-5.

9. Knauert MP, Haspel JA, Pisani MA. Sleep loss and circadian rhythm in the intensive care unit. Clin Chest Med. 2015;36:419-29.

10. Beltrami FG, Nguyen XL, Pichereau C, Maury E, Fleury B, Fagondes S. Sleep in the intensive care unit. J Bras Pneumol. 2015;41:539-46.

11. Parsons EC, Kross EK, Caldwell ES, Kapur VK, McCurry SM, Vitiello MV, et al. Post-discharge insomnia symptoms are associated with quality of life impairment among survivors of acute lung injury. Sleep Med. 2012;13:1106-9.

12. Berry R, Brooks R, Gamaldo C, Harding S, Lloyd R, Quan S, et al. A.A.S.M. scoring manual updates for 2017 (version 2.4). J Clin Sleep Med. 2017;13(5):665-6.

13. Altman MT, Knauert MP, Pisani MA. Sleep disturbance after hospitalization and critical illness: a systematic review. Ann Am Thorac Soc. 2017;14:1457-68.

14. Yang PL, Ward TM, Burr RL, Kapur VK, McCurry SM, Vitiello $\mathrm{MV}$, et al. Sleep and circadian rhythms in survivors of acute respiratory failure. Front Neurol. 2020;11:94.

15. Freedman NS, Gazendam J, Levan L, Pack AI, Schwab RJ. Abnormal sleep/wake cycles and the effect of environmental noise on sleep disruption in the intensive care unit. Am J Respir Crit Care Med. 2001;163:451-7.

16. Solverson KJ, Easton PA, Doig CJ. Assessment of sleep quality post-hospital discharge in survivors of critical illness. Respir Med. 2016;114:97-102.

17. Dhooria S, Sehgal IS, Agrawal AK, Agarwal R, Aggarwal AN, Behera D. Sleep after critical illness: study of survivors of acute respiratory distress syndrome and systematic review of literature. Indian J Crit Care Med. 2016;20(6):323-31.

18. Casey SJ, Solomons LC, Steier J, Kabra N, Burnside A, Pengo MF, Moxham J, Goldstein LH, Kopelman MD. Slow wave and R.E.M. sleep deprivation effects on explicit and implicit memory during sleep. Neuropsychology. 2016;30(8):931-45.

19. Alexopoulou C, Bolaki M, Akoumianaki E, Erimaki S, Kondili E, Mitsias P, et al. Sleep quality in survivors of critical illness. Sleep Breath. 2019;23(2):463-71.

20. Kamdar BB, Needham DM, Collop NA. Sleep deprivation in critical illness: its role in physical and psychological recovery. J Intensive Care Med. 2012;27:97-111.

21. Chishti A, Batchelor AM, Bullock RE, Fulton B, Gascoigne AD, Baudouin SV. Sleep-related breathing disorders following discharge from intensive care. Intensive Care Med. 2000;26:426-33.

22. Piva S, Fagoni N, Latronico N. Intensive care unit-acquired weakness: unanswered questions and targets for future research. F1000Res. 2019;8:508.

Publisher's Note Springer Nature remains neutral with regard to jurisdictional claims in published maps and institutional affiliations. 


\section{Authors and Affiliations}

\section{Juvva Kishan Srikanth ${ }^{1}$. Rohit Kumar ${ }^{1}$. Neeraj Kumar Gupta ${ }^{1}$ Pranav Ish ${ }^{1}$. Siddharth Raj Yadav ${ }^{1}$. Shibdas Chakrabarti ${ }^{1} \cdot$ Nitesh Gupta ${ }^{1}$}

Juvva Kishan Srikanth

drkishansrikanth@gmail.com

Rohit Kumar

dr.rohitkumar@mail.com

Neeraj Kumar Gupta

drgupta.nk@gmail.com

Pranav Ish

pranavish2512@gmail.com
Siddharth Raj Yadav

drsid28@gmail.com

Shibdas Chakrabarti

Shibdas1@yahoo.co.in

Office of Department of Pulmonary, Critical Care and Sleep Medicine, VMMC and Safdarjung Hospital, Room Number 638, Superspeciality Block, New Delhi 110029, India 